\title{
Mathematical interfaces in the
}

\section{modelling of biological populations}

\section{Michael Anthony Adena}

This thesis studies the interesting and productive interaction between mathematics and biology in the development of models of populations and their component processes. A number of detailed case studies arising from practical biological problems are developed and examined.

The major case study consists of the data analysis, sub-model construction and validation of a simulation model of the population dynamics of Australian scarab beetles. First, the biology of scarab beetles is reviewed to elucidate the most important population processes. Theoretical and empirical models of the development and survival of the subterranean immature life-stages in different soil temperatures and moistures are derived. The biological observation that low temperature fluctuations enhance development compared with a constant temperature of the mean is explained mathematically. Further theoretical results, including a useful characterisation of equivalent terperature regimes for development are also found. The environmental depression of the numbers of adult beetles caught in light traps and the sex ratio of these adult beetles at different times of the year are analysed and modelled.

Using the heat equation, a detailed sub-model of soil temperature is derived and exhaustively tested. Although a partial differential equation model for soil moisture is found to be inappropriate, a water balance approach based on the boundary conditions of the first model proves successful.

Finally, the sub-models of the life-stages and their environments are

Received 13 March 1979. Thesis submitted to the Australian National University, June 1978. Degree approved, February 1979. Supervisors: Dr A. Mazanov, Dr J.B. Gibson, Dr R.L. Davidson. 
synthesised into a complete, discrete event computer simulation model of the population dynamics of the common Australian scarab species, Sericesthis nigrolineata Boisd. at Armidale, New South Wales. This model is verified against the experimental data used to estimate its parameters, and increasingly detailed analytical models are used to verify and study the adult female behaviour and fecundity sub-model. The reasonableness of the range of the environments for which the model population can persist partially validates the final simulation model.

Three other, shorter, case studies are also analysed: the number of normal yeast colonies arising from the mating of petite yeasts; the population genetics of an interchange chromosome in rye; and statistical tests for unordered pairs in a problem in the cytogenetics of populations.

In conjunction with these three smaller studies, the simulation model of the population dynamics of scarab beetles demonstrates that rigorous, well justified solutions to complex biological problems can result from the following sequence of steps: a comprehensive review of the underlying biology; identification of the problems to be solved; formulation of formal mathematical models; analytical solution of these mathematical models frequently including computer simulation; and application of the theoretical results to the initial biological questions. The unifying power and applicability of this modular approach to the analysis and solution of problems in biology are shown to depend on the flexible use of a diversity of mathematical, statistical, and computer techniques. 